\title{
EFECTO DE LA SUSTITUCIÓN PARCIAL DE LA HARINA DE TRIGO POR TORTA DE SACHA INCHI (Plukenetia volubilis L.) EN LAS PROPIEDADES REOLÓGICAS DE LA MASA DE PAN DULCE
}

\author{
EFFECT OF THE PARTIAL REPLACEMENT OF WHEAT FLOUR BY CAKE \\ OF SACHA INCHI (Plukenetia volubilis L.) ON THE RHEOLOGICAL \\ PROPERTIES OF DOUGH IN SWEET BREAD
}

\author{
${ }^{1}$ Angel Dionel Toralva Aylas, ${ }^{1}$ Maribel Rodas Pingus, ${ }^{2}$ Dániza Mirtha Guerrero Alva
}

\section{RESUMEN}

El presente estudio tuvo por finalidad la evaluación del efecto de la sustitución parcial de la harina de trigo (HT) por torta de sacha inchi (TSI) sobre las propiedades reológicas de una masa de pan dulce así como las propiedades sensoriales de los bizcochos obtenidos. Se realizaron tres tratamientos y un control (T1, 100\%HT; T2, 90\%HT/10\%TSI; T3, $80 \% \mathrm{HT} / 20 \% \mathrm{TSI}$ y T4, $70 \% \mathrm{HT} / 30 \% \mathrm{TSI})$ a los que se les determinó las propiedades reológicas utilizando los equipos alveógrafo y consistógrafo. Se produjeron los bizcochos y se evaluaron las propiedades sensoriales de la mezcla (HT/TSI) con mejor comportamiento reológico, así mismo se realizaron análisis proximales, físicos y químicos de dichos productos. Los resultados del estudio reológico de la masa de pan dulce con cada mezcla de harina de trigo-torta de sacha inchi mostraron un mejor comportamiento en la mezcla T2. Por otro lado la evaluación sensorial (T1, 100\% HT; T2, 90\% HT/10\% TSI), muestra buena aceptabilidad, y la composición proximal (humedad, cenizas, proteína, grasa, fibra y carbohidratos) fue mejor para el tratamiento T2 así como las propiedades físicas (peso, volumen, volumen específico) y química ( acidez total) no presentaron diferencias

Palabras clave: Harina de trigo/Torta de sacha inchi, propiedades reológicas, pan dulce.

\section{ABSTRACT}

The present study was aimed at evaluating the effect of the partial substitution of wheat flour (WF) by sachai Inchi cake (SIC) on the rheological properties of dough and sensory properties of the sweet bread obtained. Three treatments and a control (T1, 100\%WF; T2, WF90\%/10\%SIC; T3, 80\%WF/20\%SIC and T4, 70\%WF/30\%SIC) were made which were determined rheological properties using a consistograph and an alveograph. Sweet bread was produced and the sensory properties of the mixture (WF/SIC) with improved rheological behavior was evaluated, likewise proximal, physical and chemical analysis of the products were performed. The results of the rheological study of sweet bread dough with each mix wheat flour/sacha inchi cake showed a better performance in the T2 mixture. On the other hand sensory evaluation (T1,100\%WF; T2, 90\%WF/10\% SIC) show good acceptability, and the proximate composition (moisture, ash, protein, fat, fiber and carbohydrates) was best for T2 treating and physical (weight, volume, specific volume) and chemical properties (total acidity) did not differ.

Keywords: wheat flour/sacha inchi cake, rheological properties, sweet bread.

\section{INTRODUCCIÓN}

Los productos de panificación, debido a sus características de vida en anaquel (baja $\mathrm{a}_{\mathrm{w}}$ ), son menos susceptibles al crecimiento microbiano. Por su bajo costo y formulación, son productos que pueden ser enriquecidos al adicionar otras proteínas, como concentrados o aislados proteicos de diversas leguminosas (Güemes-Vera, 2009). Las proteínas son escasas en la mayoría de los países en vías de desarrollo y además, por ser las más costosas de producir son las más difíciles de adquirir debido al alto índice de crecimiento demográfico.

Se realizan diversas investigaciones sobre el uso de proteínas no convencionales para el consumo humano con 
Toralva, A. et al. Efecto de la sustitución parcial de la harina de trigo por torta de sacha inchi en las propiedades reológicas de la masa de pan dulce.

el fin de poder satisfacer las necesidades de este nutrimento en las poblaciones de pocos recursos (Badui, 1990). Una adecuada mezcla de alimentos vegetales puede satisfacer los requerimientos de todos los aminoácidos necesarios (Montgomery, 1998). Esto significa una complementación aminoacídica eficaz. Un producto adecuado para este tipo de mezclas de cereales y leguminosas es el pan dulce conocido como bizcocho.

En el Perú contamos con el sacha inchi (Plukenetia volubilis L.), un producto de la selva que tiene una creciente inclusión en la alimentación con grandes bondades nutricionales (Tito, 2009). Se ha demostrado que las semillas de
Plukenetia volubilis L, tienen alto contenido de proteínas $(33 \%)$ y aceite $(49 \%)$ (Tito, 2009$)$. En las empresas agroindustriales solo se aprovecha el aceite dejando de lado la torta de sacha inchi, rica en proteínas y restos de ácidos grasos $\omega_{3}$. Las proteínas son responsables en gran medida de la textura y de las características reológicas de muchos alimentos, y las alteraciones indeseables físicas o químicas que estos sufren dan como resultado una calidad sensorial y nutricional pobre que lleva consigo el rechazo del producto (Badui, 1990). Existen alimentos cuya textura está determinada por las interacciones físicas y químicas de las proteínas con el almidón. Las propiedades funcionales de las proteínas en los

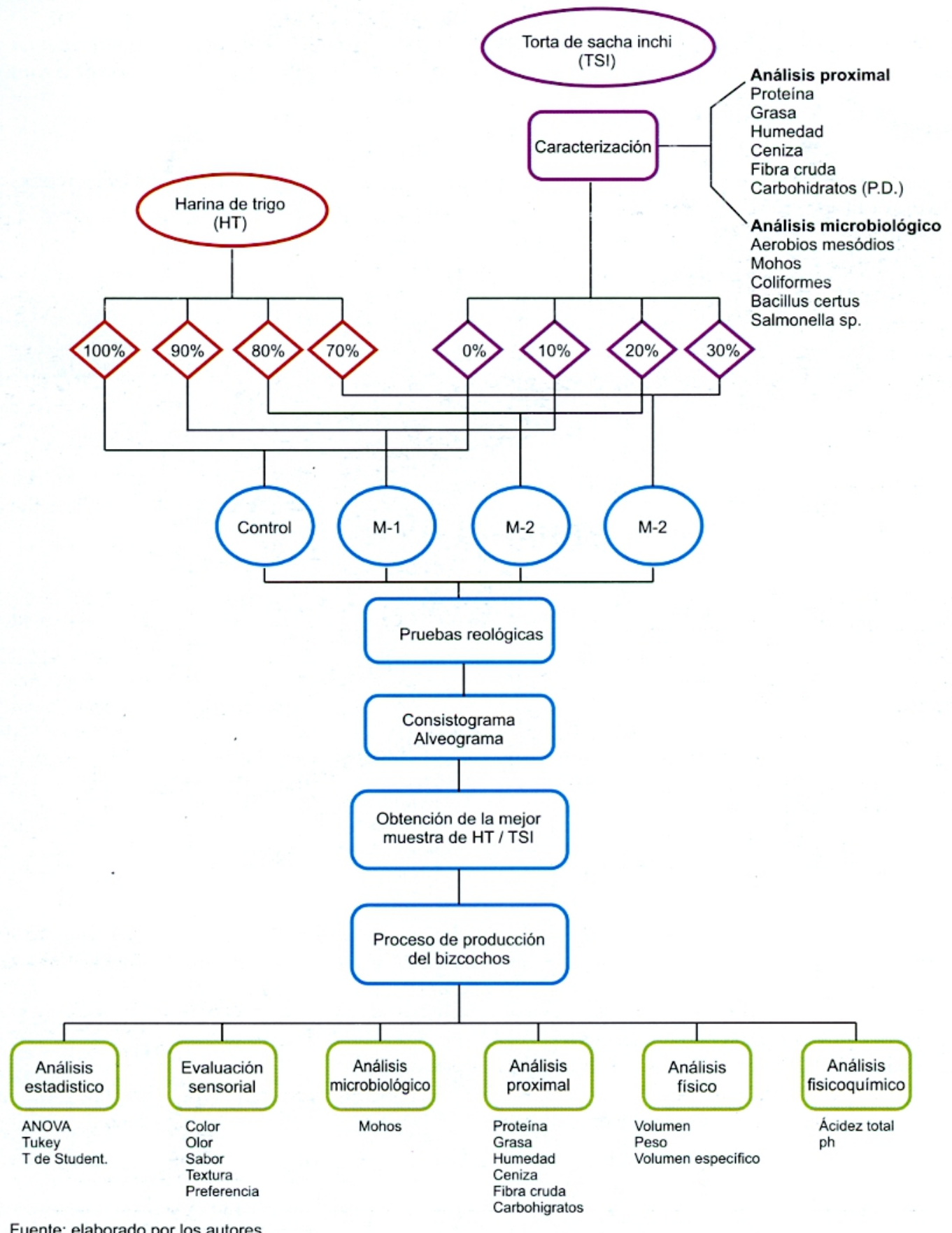

Figura 1. Diagrama experimental del estudio 
Toralva, A. et al. Efecto de la sustitución parcial de la harina de trigo por torta de sacha inchi en las propiedades reológicas de la masa de pan dulce.

alimentos están relacionadas con sus características estructurales y físico-químicas. Para mejorar el comportamiento de las proteínas en los alimentos, es fundamental conocer las propiedades físicas, químicas, nutritivas y funcionales de las proteínas y los cambios sufridos durante el procesado (Fennema, 2000). La presente investigación pretende aprovechar la torta de sacha inchi en la sustitución de harina de trigo en la elaboración de bizcocho, que es un pan dulce muy consumido en nuestro país.

\section{MATERIALES Y MÉTODOS}

\section{Materiales}

Se utilizó la torta de sacha inchi (mezcla de las variedades Pinto Recodo, Tambo Yaguas, Muyuy, Cumbaza, Lamas, Shanao y Río Putumayo).

\section{Equipospara reologia: alveógrafo y consistógrafo}

\section{Metodologia del trabajo experimental:}

El trabajo de investigación se desarrolló mediante un diseño experimental. (Figura 1).

La caracterización de la torta de sacha inchi, se hizo siguiendo los métodos descritos por la AOAC 1990 y los análisis microbiológicos se efectuaron según la normativa peruana de criterios microbiológicos.

Para la evaluación de las propiedades reológicas, se utilizó mezclas de harina de trigo y torta de sacha inchi $(\mathrm{Plu}$ kenetia volubilis L.) con sustituciones de $10 \%, 20 \%$ y $30 \%$, y un control sin sustitución, que se analizaron en el alveógrafo mediante el método AACC 54-30 (2000) y en el consistógrafo por el método AACC 54-50 (2000).

La producción de los bizcochos se realizó en las

Tabla 1. Resultados de los análisis proximales de la torta de sacha inchi (Plukenetia volubilis L.)

\begin{tabular}{lcccc}
\hline \multicolumn{5}{c}{ Muestras } \\
Componentes (\%) & $\mathbf{1}$ & $\mathbf{2}$ & $\mathbf{3}$ & $\dot{\mathbf{X}}+/-\mathrm{DS}$ \\
\hline Humedad & 5,152 & 5,221 & 5,176 & $5,183+/-0,35$ \\
Proteína & 51,239 & 51,214 & 51,246 & $51,233+/-0,17$ \\
Grasa & 5,782 & 5,748 & 5,621 & $5,717+/-0,08$ \\
Fibra Cruda & 4,681 & 4,751 & 4,642 & $4,691+/-0,06$ \\
Cenizas & 4,154 & 4,713 & 4,173 & $4,347+/-0,32$ \\
Carbohidratos & 28,460 & 28,893 & 29,142 & $28,832+/-0,35$ \\
\hline
\end{tabular}

Tabla 3. Datos obtenidos mediante consistógrafo de las mezclas con sustitución de la harina de trigo por torta de sacha inchi (Plukenetia volubilis L.)

\begin{tabular}{lcccc}
\hline \multicolumn{1}{c}{ Características } & \multicolumn{4}{c}{ Sustitución (\% TSI) } \\
& Control (HT) & $\mathbf{1 0 \%}$ & $\mathbf{2 0 \%}$ & $\mathbf{3 0 \%}$ \\
\hline HYDHA b 15 (\%) & 56,8 & 52,55 & 46,0 & 36,1 \\
PrMax (mb) & 2277 & 2132 & 2180 & 2103 \\
TPrMax (s) & 120 & 140 & 131 & 150 \\
Tol (s) & 188 & 172 & 158 & 188 \\
D250 (mb) & 478 & 440 & 560 & 358 \\
D450 (mb) & 941 & 912 & 1114 & 886 \\
WAC b 15 \% & 59,7 & 54,6 & 48,4 & 38,2 \\
\hline HT=Harina de trigo & & & &
\end{tabular}

$\mathrm{HT}=$ Harina de trigo

TSI $=$ Torta de sacha inchi instalaciones del Centro Experimental Tecnológico (CET), empleando el Método Directo.

Los análisis realizados a los bizcochos fueron: análisis proximal según los métodos descritos por la AOAC 1990 , análisis físicos según Da Mota (2005), acidez total según la NTP 206.008 (Revisada el 2011) y para la evaluación sensorial se utilizó la prueba de preferencia con escala hedónica de 5 puntos $(1=$ me disgusta mucho a $5=$ Me gusta mucho $)$, a través de los ítems sabor, textura, color, olor y aceptabilidad del producto.

\section{RESULTADOS}

\section{Caracterización de la torta de sacha inchi.}

En las tablas 1 y 2 se muestran los resultados de los valores obtenidos para la caracterización de la torta de sacha inchi.

\section{Análisis reológico}

La evaluación de las propiedades reológicas de la mezcla de harina de trigo y torta de sacha inchi se muestran en las tablas 3 y 4 .

\section{Análisis proximal de los bizcochos}

En la tabla 5 se muestra los resultados del análisis proximal de los bizcochos con $10 \%$ de sustitución de torta de sacha inchi en comparación con un control de harina de trigo.

\section{Análisis físico y químico}

En las tablas 6 y 7 se muestran los resultados obtenidos referentes al volumen, peso, volumen específico y acidez de las muestras analizadas.

Tabla 2. Resultados de los análisis microbiológicos de la torta de sacha inchi (Plukenetia volubilis L.)

\begin{tabular}{lcccc}
\hline \multicolumn{5}{c}{ Muestras } \\
\multicolumn{1}{c}{ Análisis } & $\mathbf{1}$ & $\mathbf{2}$ & $\mathbf{3}$ & $\dot{\mathbf{X}}+/$-DS \\
\hline Aerobios & $2,4 \times 10^{3}$ & $1,8 \times 10^{3}$ & $2,3 \times 10^{3}$ & $2,2 \times 10^{3}+/-$ \\
mesófilos & $\mathrm{UFC} / \mathrm{g}$ & $\mathrm{UFC} / \mathrm{g}$ & $\mathrm{UFC} / \mathrm{g}$ & $0,32 \times 10^{3} \mathrm{UFC} / \mathrm{g}$ \\
Mohos & $<100 \mathrm{UFC} / \mathrm{g}$ & $<100 \mathrm{UFC} / \mathrm{g}$ & $<100 \mathrm{UFC} / \mathrm{g}$ & $<100 \mathrm{UFC} / \mathrm{g}$ \\
Coliformes & $93 \mathrm{NMP} / \mathrm{g}$ & $93 \mathrm{NMP} / \mathrm{g}$ & $75 \mathrm{NMP} / \mathrm{g}$ & $\begin{array}{c}87+/-0,01 \\
\mathrm{NMP} / \mathrm{g}\end{array}$ \\
Bacillus cereus & $<100 \mathrm{UFC} / \mathrm{g}$ & $<100 \mathrm{UFC} / \mathrm{g}$ & $<100 \mathrm{UFC} / \mathrm{g}$ & $<100 \mathrm{UFC} / \mathrm{g}$ \\
Salmonella sp. & Ausencia & Ausencia & Ausencia & Ausencia \\
\hline
\end{tabular}

Tabla 4. Resultados obtenidos con el alveógrafo al sustituir la harina de trigo por torta de sacha inchi (Plukenetia volubilis L.)

\begin{tabular}{lcccc}
\hline \multicolumn{1}{c}{ Características } & \multicolumn{4}{c}{ Sustitución (\%TSI) } \\
& Control (HT) & $\mathbf{1 0 \%}$ & $\mathbf{2 0} \%$ & $\mathbf{3 0 \%}$ \\
\hline Tenacidad P (mm) & $80^{\mathrm{a}}$ & $94^{\mathrm{a}}$ & $135^{\mathrm{b}}$ & $197^{\mathrm{c}}$ \\
Extensibilidad L (mm) & $112,1^{\mathrm{a}}$ & $67,7^{\mathrm{b}}$ & $31,4^{\mathrm{c}}$ & $11,8^{\mathrm{d}}$ \\
Fucrza W (10E-4J) & $321^{\mathrm{a}}$ & $253^{\mathrm{b}}$ & $198^{\mathrm{c}}$ & $133^{\mathrm{d}}$ \\
Índice de equilibrio (P/L) & $0,71^{\mathrm{a}}$ & $1,39^{\mathrm{a}}$ & $4,36^{\mathrm{b}}$ & $17,3^{\mathrm{c}}$ \\
\hline
\end{tabular}

El superíndice que aparece en los promedios de cada parámetro indica la diferencia significativa según la ANOVA (prueba de Tukey, $\mathrm{p}<0,05$ )

$\mathrm{HT}=$ Harina de trigo

$\mathrm{TSI}=$ Torta de sacha inch 
Toralva, A. et al. Efecto de la sustitución parcial de la harina de trigo por torta de sacha inchi en las propiedades reológicas de la masa de pan dulce.

Tabla 5. Composición proximal de los bizcochos según el nivel de sustitución de torta de sacha inchi (Plukenetia volubilis L.)

\begin{tabular}{lcc}
\hline Componentes & $\begin{array}{c}\text { Nivel de sustitución de torta de sacha inchi } \\
\text { Control (HT) } \\
\dot{\mathbf{X}}+/-\mathrm{DS}\end{array}$ & $\begin{array}{c}\mathbf{1 0} \% \text { (TSI) } \\
\dot{\mathbf{X}}+/-\mathrm{DS}\end{array}$ \\
\hline Humedad & $23,81+/-0,40$ & $25,58+/-0,33$ \\
Proteína & $10,28+/-0,16$ & $11,74+/-0,28$ \\
Grasa & $6,30+/-0,10$ & $8,16+/-0,03$ \\
Fibra cruda & $0,20+/-0,10$ & $0,35+/-0,04$ \\
Cenizas & $1,03+/-0,39$ & $1,51+/-0,08$ \\
Carbohidratos & $58,38+/-0,17$ & $52,66+/-0,11$ \\
Energía & $331,38+/-0,94$ & $331,01+/-0,96$ \\
\hline
\end{tabular}

$\mathrm{HT}=$ Harina de trigo

$\mathrm{TSI}=$ Torta de sacha inchi

Tabla 7. Determinación de acidez total en bizcochos según la sustitución de torta de sacha inchi (Plukenetia volubilis L.)

\begin{tabular}{lcc}
\hline \multicolumn{1}{c}{ Análisis } & \multicolumn{2}{c}{ Muestras } \\
& Control (HT) & $\mathbf{1 0 \%}$ (TSI) \\
& $\dot{\mathbf{X}}+/$-DS & $\dot{\mathbf{X}}+/-\mathrm{DS}$ \\
\hline Acidez total $(\mathrm{g} / 100 \mathrm{~g}$ expresado en & $0,094+/-0,001$ & $0,094+/-0,001$ \\
ácido sulfúrico) & & \\
\hline
\end{tabular}

HT $=$ Harina de trigo

TSI $=$ Torta de sacha inch

Tabla 9. Respuestas según la escala hedónica de aceptabilidad para cada nivel de sustitución de los bizcochos con torta de sacha inchi (Plukenetia volubilis L.)

\begin{tabular}{lcccc}
\hline $\begin{array}{c}\text { Escala hedónica de } \\
\text { aceptabilidad } \\
\text { (valoración) }\end{array}$ & $\begin{array}{c}\text { Nivel de sustitución de torta de sacha inchi } \\
\text { Control (HT) }\end{array}$ & $\begin{array}{c}\text { 10\% } \\
\text { (TSI) }\end{array}$ \\
\hline Cantidad & Porcentaje Cantidad Porcentaje \\
Me gusta mucho (5) & 2 & 6,7 & 0 & 0 \\
Ni me gusta ni me & 25 & 83,3 & 3 & 10 \\
disgusta (3) & 3 & 10 & 18 & 60 \\
Me disgusta (2) & 0 & 0 & 7 & 23,3 \\
Me disgusta mucho (1) & 0 & 0 & 2 & 6,7 \\
Total & 30 & 100 & 30 & 100 \\
\hline
\end{tabular}

$\mathrm{HT}=$ Harina de trigo

TSI $=$ Torta de sacha inchi

\section{Análisis microbiológicos}

En la tabla 8 se muestra el resultado de la verificación de la calidad sanitaria de los bizcochos según la sustitución con torta de sacha inchi.

\section{Análisis sensorial}

En las tablas 9 y 10 se muestran los resultados de la prueba de preferencia con escala hedónica de 5 puntos y organolépticas.

\section{DISCUSIÓN}

La torta de sacha inchi analizada por Mondragón (2009) presentó menor contenido de proteína 37,33g/100g en comparación con lo obtenido en esta investigación que fue de $51,233+/-0,17 \mathrm{~g} / 100 \mathrm{~g}$. En cuanto al contenido de fibra bruta el mismo autor citado reportó $3,16 \mathrm{~g} / 100 \mathrm{~g}$ cantidad menor a nuestro resultado que fue de 4,691+/$0,06 \mathrm{~g} / 100 \mathrm{~g}$. Respecto al contenido de carbohidratos Mon-
Tabla 6. Determinación de las características físicas del bizcocho según la sustitución de torta de sacha inchi (Plukenetia volubilis $\mathrm{L}$.)

\begin{tabular}{lcc}
\hline \multicolumn{1}{c}{ Análisis } & \multicolumn{2}{c}{ Muestra } \\
& Control (HT) & $\mathbf{1 0 \%}$ (TSI) \\
& $\dot{\mathbf{X}}+/$-DS & $\dot{\mathbf{X}}+/$-DS \\
\hline Volumen $\left(\mathrm{cm}^{3}\right)$ & $233,83+/-14,45$ & $200,08+/-8,54$ \\
Peso $(\mathrm{g})$ & $43,67+/-1,52$ & $46,33+/-0,58$ \\
Volumen especifico $\left(\mathrm{cm}^{3} / \mathrm{g}\right)$ & $5,35+/-0,16$ & $4,31+/-0,19$ \\
\hline
\end{tabular}

$\mathrm{HT}=$ Harina de trigo

TSI $=$ Torta de sacha inchi

Tabla 8. Análisis microbiológico de bizcochos según el nivel de sustitución de torta de sacha inchi (Plukenetia volubilis L.)

\begin{tabular}{lcccc}
\hline Análisis & Sustitución de TSI & \multicolumn{3}{c}{ Valor Normado } \\
& Control & $10 \%$ & Mínimo & Máximo \\
\hline Mohos & $<10 \mathrm{UFC} / \mathrm{g}$ & $<10 \mathrm{UFC} / \mathrm{g}$ & $10^{2}$ & $10^{3}$ \\
\hline
\end{tabular}

UFC: Unidades formadoras de colonias

TSI: Torta de sacha inchi

Tabla 10. Promedio y desviación estándar de la prueba sensorial hedónica según el nivel de sustitución de torta de sacha inchi en el bizcocho.

\begin{tabular}{lccc}
\hline \multicolumn{1}{c}{ Características } & $\begin{array}{c}\text { Nivel de sustitución de TSI } \\
\text { Control (0\%) } \\
\dot{\mathbf{X}}+/-\mathbf{D S}\end{array}$ & $\begin{array}{c}\text { Valor } \mathbf{p} \\
\mathbf{1 0} \%\end{array}$ \\
\hline Sabor & $4,23+/-0,63$ & $2,63+/-0,81$ & 0,08 \\
Olor & $3,97+/-0,67$ & $2,37+/-0,960,02$ \\
Color & $3,93+/-0,74$ & $3,30+/-0,920,04$ \\
Textura & $3,93+/-0,78$ & $3,53+/-0,90$ & 0,22 \\
\hline
\end{tabular}

TSI: Torta de sacha inchi

*Existe diferencia significativa

dragón obtuvo $22,01 \mathrm{~g} / 100 \mathrm{~g}$, menor a la encontrada en este trabajo de $28,832+/-0,35 \mathrm{~g} / 100 \mathrm{~g}$, pero la diferencia mayor se halló en el contenido de grasa donde Mondragón obtuvo $37,33 \mathrm{~g} / 100 \mathrm{~g}$, y en la presente investigación $5,717+/$ $0,08 \mathrm{~g} / 100 \mathrm{~g}$, valores semejantes a los obtenidos por Pascual et al. (2000) y Hurtado (2013), cuyos valores fueron $6,93 \mathrm{~g} / 100 \mathrm{~g}$ y $4,84 \mathrm{~g} / 100 \mathrm{~g}$ respectivamente.

En el análisis microbiológico de la torta de sacha inchi se expresa que el producto cumple con las condiciones adecuadas para la elaboración de los bizcochos de acuerdo a la Norma Técnica de Salud $\mathrm{N}^{\circ} 071$.

Respecto a las pruebas reológicas, en los análisis en el alveograma, Escobar y Varela (2008) obtuvieron una mezcla de harina de trigo y harina de papa criolla que presentó un adecuado comportamiento panadero de las mezclas con valores del índice $\mathrm{P} / \mathrm{L}$ de 1,54 y W de 233; resultados semejantes encontramos en esta investigación para la mezcla con $10 \%$ de sustitución con torta de sacha inchi, la cual presentó un índice de $\mathrm{P} / \mathrm{L}$ de $1,39, \mathrm{y} \mathrm{W}$ de 253 , siendo el valor de $\mathrm{P} / \mathrm{L}$ 
Toralva, A. et al. Efecto de la sustitución parcial de la harina de trigo por torta de sacha inchi en las propiedades reológicas de la masa de pan dulce.

del control 0,71 y 321 para W, valores que indican un buen comportamiento panadero. Con respecto a las otras mezclas con $20 \%$ y $30 \%$ de sustitución con torta de sacha inchi, éstas presentaron un índice de $\mathrm{P} / \mathrm{L}$ de 4,36 y 11,8 y valores de W de 198 y 133 respectivamente, resultados que nos indican que dichas mezclas no tienen un buen comportamiento panadero; debido posiblemente a la mayor presencia de grasa y proteínas propias de la sustitución.

En los análisis realizados en el consistógrafo, Escobar y Varela (2008) obtuvieron una mezcla de harina de trigo y harina de papa criolla con una capacidad de absorción de agua de 55,5\% y una consistencia de la masa apropiada, la resistencia a la presión (PrMax) de hasta 3158 mbar; resultados similares se obtuvieron en la presente investigación para la mezcla control y la muestra con $10 \%$ de torta de sacha inchi presentando una capacidad de adsorción de agua de $56,8 \%$ y $52,5 \%$ y la resistencia a una presión de 2277 mbar y $2132 \mathrm{mbar}$ respectivamente, valores que nos indican que dichas mezclas darán una consistencia de masa apropiadas; las otras mezcla con $20 \%$ y $30 \%$ de sustitución con torta de sacha inchi tuvieron una capacidad de absorción de agua de $46,0 \%$ y $36,1 \%$ y resistencia a una presión de $2180 \mathrm{mbar}$ y $2103 \mathrm{mbar}$ respectivamente. Esto nos indica que las muestras con $20 \%$ y $30 \%$ de sustitución de sacha inchi no son aptas para la panificación.

Con relación a los análisis proximales Arroyave y Esguerra (2006) obtuvieron productos de panificación utilizando quinua que presentaron nivel de proteínas de hasta de $10 \mathrm{~g} / 100 \mathrm{~g}$. Mejores resultados se obtuvieron en la presente investigación, alcanzando niveles de proteína de $11,74 \mathrm{~g} / 100 \mathrm{~g}$ en bizcochos con $10 \%$ de sustitución de torta de sacha inchi; además, el contenido de grasa fue de 8,16 $\mathrm{g} / 100 \mathrm{~g}$ en la muestra con $10 \%$ de torta de sacha inchi $\mathrm{y}$ $6,30 \mathrm{~g} / 100 \mathrm{~g}$ de grasa en el bizcocho control, lo que se debería al aporte de grasa por parte de la torta de sacha inchi.

Con respecto a los análisis físicos del bizcocho se obtuvieron diferencias entre la muestra control y la muestra con sustitución de $10 \%$ de torta de sacha inchi; el peso de la muestra control fue en promedio $44,4 \mathrm{~g}$ y $46,1 \mathrm{~g}$ en la muestra con $10 \%$ de torta de sacha inchi, debido posiblemente a la mayor retención de agua por presencia de las proteínas de sacha inchi. Con relación al volumen se encontró $233,63 \mathrm{~cm}^{3}$ para el control y $200,08 \mathrm{~cm}^{3}$ en el bizcocho con la adición de torta de sacha inchi, lo que ocurre cuando en la fórmula se reduce el contenido de gluten. En los análisis de acidez total no se encontró diferencias significativas entre la muestra control y la muestra con sustitución de $10 \%$ de torta de sacha inchi.

Con respecto a la prueba de aceptabilidad Aire y Taipe (2011) elaboraron bebidas a base de torta de sacha inchi con tres tipos de diluciones $(1: 4,1: 4,5$ y $1: 5)$ sacha inchi: agua y un control de bebida de soya, encontrando que la bebida con disolución 1:4,5 (sacha inchi: agua) fue la más aceptada; comparando con los resultados obtenidos en la presente investigación los panelistas indicaron que sí hallaron diferencia significativa entre los bizcochos control y con sustitución de $10 \%$ de torta de sacha inchi aplicando la prueba de $T$ de Student $(\mathrm{p}<0,05)$, esto puede deberse a la falta de costumbre que existe en el consumo de sacha inchi.

En relación a la prueba organoléptica Aire y Taipe
(2011), encontraron diferencias significativas con respecto al sabor y olor aplicando la prueba de ANVA $(p<0,05)$. La presente investigación también obtuvo diferencias significativas en las características organolépticas de olor entre los bizcochos control y con $10 \%$ de sustitución de torta de sacha inchi, similar al encontrado en la bebida a base de torta de sacha inchi; asimismo se hallaron diferencias significativas respecto al color entre los bizcochos control y con $10 \%$ de sustitución de torta de sacha inchi, lo que puede explicarse a que la torta de sacha inchi confirió al producto un color más intenso. Sin embargo, en el presente estudio no se encontró diferencias significativas en el sabor y la textura, esto puede deberse a que en el proceso de panificación, la adición de la torta de sacha inchi confirió mejor textura y sabor a los productos.

\section{CONCLUSIONES}

La caracterización de la torta de sacha inchi (mezcla de las variedades Pinto Recodo, Tambo Yaguas, Muyuy, Cumbaza, Lamas, Shanao y Río Putumayo) tuvo la siguiente composición: humedad $5,18 \%$, proteína $51,23 \%$, grasas $5,71 \%$, fibra cruda $4,69 \%$, cenizas $4,34 \%$ y carbohidratos $28,83 \%$.

Con el consistógrafo se observó una hidratación equivalente de $52,55 \%$ y presión máxima de $2132 \mathrm{mb}$; mientras que el tiempo para llegar al pico de presión máxima fue de 140 segundos, y el tiempo que la curva permaneció estable fue de 188 segundos, el debilitamiento de la curva a 250 segundos y 450 segundos fue de $440 \mathrm{mb}$ y $912 \mathrm{mb}$. Con el alveógrafo la relación $\mathrm{P} / \mathrm{L}$ fue de 1,39 para un buen comportamiento panadero, la tenacidad fue de $94 \mathrm{~mm}$, la extensibilidad fue de $253 \mathrm{~mm}$ y la fuerza $253 \times 10-^{4} \mathrm{~J}$, valores que corresponden a un mezcla con características panaderas, siendo el porcentaje óptimo de sustitución de $10 \%$ de torta de sacha inchi.

La evaluación sensorial descriptiva de los bizcochos con $10 \%$ de sustitución de torta de sacha inchi fue: aspecto externo: menor volumen y corteza con color más intenso respecto al control, aroma a sacha inchi. Aspecto interno: miga color beige intenso y más compacto respecto al control, sabor dulce y textura suave.

La composición proximal del producto con $10 \%$ de sustitución de torta de sacha inchi fue: humedad 25,58\%, proteína $11,74 \%$, grasa $8,15 \%$, fibra cruda $0,35 \%$, ceniza $1,51 \%$, carbohidratos $52,66 \%$ y energía $331,01 \mathrm{Kcal}$.

Los productos procesados con $10 \%$ de torta de sacha inchi cumplieron con los criterios microbiológicos para los productos de su tipo, mientras que en la evaluación sunsorial los panelistas demostraron agrado y un amplio porcentaje no mostraron rechazo, hecho que puede ser dirigido a su consumo, por la composición proximal del producto y valor nutricional del sacha inchi.

\section{REFERENCIAS BIBLIOGRÁFICAS}

Abascal de la Vega, L. (2005). Propuesta de Elaboración de Pan Blanco Fortificado con zinc para el consumo de Pacientes en el Hospital Roosevelt (tesis de pregrado). Universidad de San Carlos de Guatemala, Gua- 
Toralva, A. et al. Efecto de la sustitución parcial de la harina de trigo por torta de sacha inchi en las propiedades reológicas de la masa de pan dulce.

temala.

Aire, Y. y Taipe, K. (2011). Elaboración y caracterización de bebida esterilizada a partir de sacha Inchi (Plukenetia volubilis L.) (tesis de pregrado). Universidad Nacional Daniel Alcides Carrión, Chanchamayo, Perú

Arroyave, L. \& Esguerra, C. (2006). Utilización de la harina de quinua (Chenopodium quinoa $W$ ild) en el proceso de panificación (Tesis de pre grado). Universidad de la Salle, Bogotá, Colombia.

Badui, S. (1990). Quimica de los alimentos. México DF: Editorial Alhambra Mexicana S.A. Segunda Edición.

Baños, B. (2007). Caracterización reológicas de masas de harina de trigo adicionadas con fibra soluble (tesis de pregrado). Universidad Autónoma del Estado de Hidalgo. Tulancingo de Bravo, México.

Calaveras, J. (2004). Nuevo tratado de panificación y bolleria.
Madrid: Editorial Mundi-Prensa. Segunda Edición.

Carpenter, R., Lyon, D. \& Hasdell, T. (2002). Análisis sensorial en el desarrollo y control de la calidad de alimentos. Zaragoza, España: Acribia Editorial S.A. Segunda Edición.

Cegarra, J. (2004). Metodología de la investigación cientifica y tecnológica. Madrid, España: Editorial Díaz de Santos S.A.

Cepeda, R y Corchuelo, G. (1991). Tecnología de cereales y oleaginosas. Bogotá, Colombia: Ministerio de educación nacional. Unidad universitaria del sur de Bogotá.

REVISTA VIRTUAL PRO. (2011) Algunas operaciones unitarias aplicadas en la industria de alimentos. Virtual pro, Vol. 11 (117): 100.

Rodriguez, E., Fernández, A., \& Ayala, A. (2005). Modelos reológicos aplicados a masas de trigo y maiz. Ingeniería Investigación, 25(2), 87-93.

\section{Correspondencia:}

Ángel Dionel Toralva Aylas: angeldioneltoralva@hotmail.com

Maribel Rodas Pingus: maribelrodas24@hotmail.com
Fecha de Recepción: 13/10/2015

Fecha de Aceptación: 02/12/2015 